
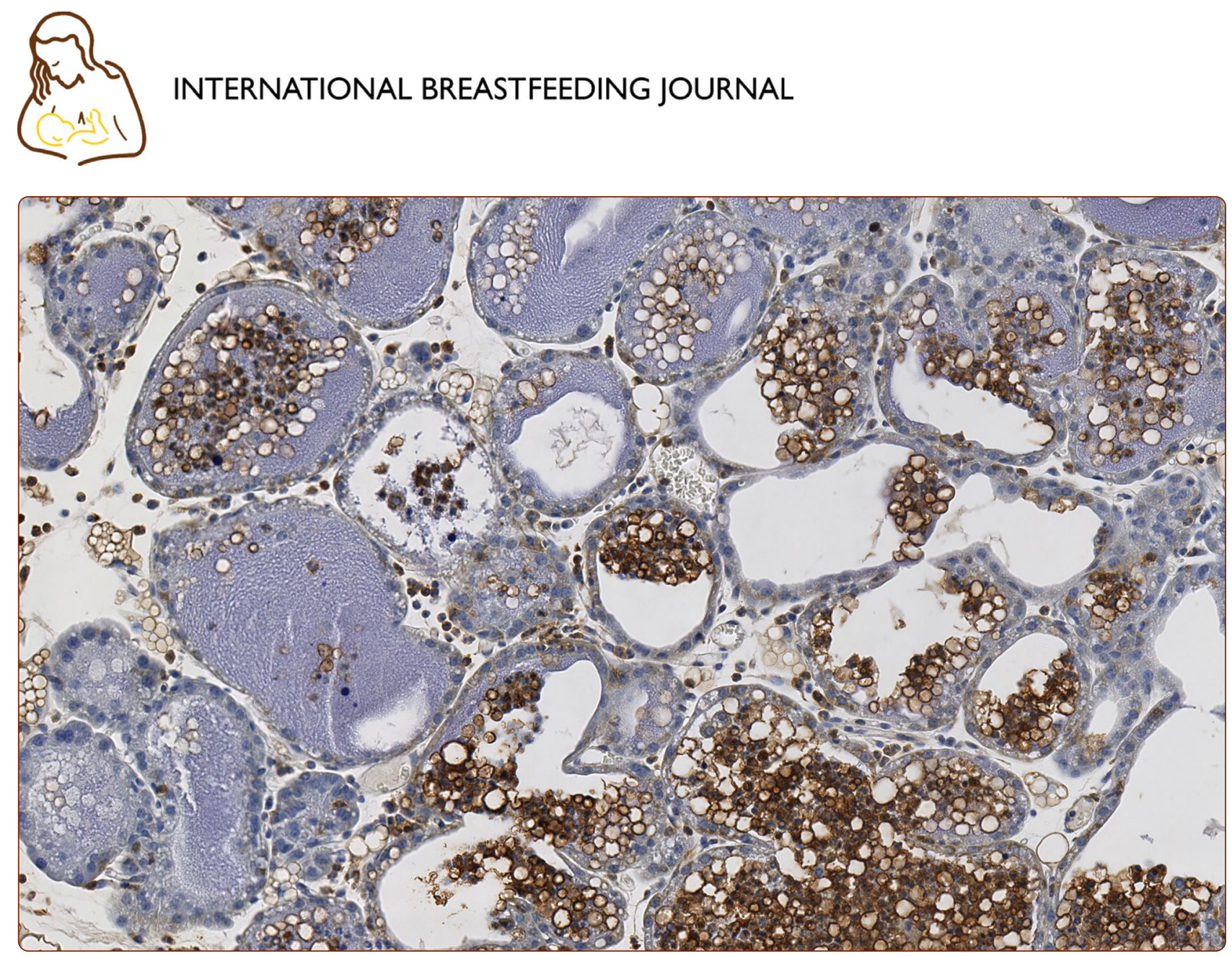

\title{
Mouse models of mastitis - how physiological are they?
}

Ingman et al. 


\title{
Mouse models of mastitis - how physiological are they?
}

Wendy V Ingman ${ }^{1,2^{*}}$, Danielle J Glynn ${ }^{1,2}$ and Mark R Hutchinson ${ }^{3}$

\begin{abstract}
Lactation mastitis is a common, but poorly understood, inflammatory breast disease that is a significant health burden. A better understanding of the aetiology of mastitis is urgently required, and will assist in the development of improved prevention and treatment strategies in both human and animal species. Studies in mice have the potential to greatly assist in identifying new drug candidates for clinical trials, and in developing a better understanding of the disease. Mouse models of mastitis involve administration of a mastitis-inducing agent to the mammary gland usually during lactation to examine the host immune response, and progression through to resolution of the disease. There are important variations in the protocols of these mouse models that critically affect the conclusions that can be drawn from the research. Some protocols involve weaning of offspring at the time of mastitis induction, and there are variations in the mastitis-inducing agent and its carrier. Induction of mammary gland involution through weaning of offspring limits the capacity to study the disease in the context of a lactating mammary gland. Administration of live bacteria in an aqueous carrier can cause sepsis, restricting the physiological relevance of the model. Mouse model research should employ appropriately designed controls and closely monitor the health of the mice. In this commentary, we discuss the advantages and study design limitations of each mouse model, and highlight the potential for further development of physiologically relevant mouse models of mastitis.
\end{abstract}

Keywords: Mastitis, Mouse models, Inflammation, Infection, Involution, Mammary gland

\section{Background}

Mastitis is a common, but poorly understood, inflammatory breast disease in lactating women that causes localised pain, reduced milk synthesis and the rapid onset of systemic symptoms including fever, flu-like aches, chills and fatigue [1,2]. The challenges posed by this disease lead many women to use supplementary formula, or cease breastfeeding altogether [3-5]. Mastitis in HIVinfected women increases the risk of vertical transmission of HIV to the breastfeeding infant [6,7].

The cause of mastitis is believed to be infection of the breast with bacterial pathogens [8,9]. However, recent research suggests this paradigm might be an oversimplification [10,11]. Milk stasis and maternal stress are strong predisposing factors, and alternative microorganisms such as commensal bacteria [12] and fungi [13] have also been implicated, all of which may trigger or

\footnotetext{
* Correspondence: wendy.ingman@adelaide.edu.au

'Discipline of Surgery, School of Medicine, The Queen Elizabeth Hospital, University of Adelaide, Woodville, Australia

${ }^{2}$ Robinson Research Institute, University of Adelaide, Adelaide, Australia Full list of author information is available at the end of the article
}

amplify breast inflammation leading to mastitis $[14,15]$. Therefore, the interactions between inflammatory stimuli including pathogenic bacteria [8] and other components of the microbiome, as well as the host immune response $[15,16]$ are all likely to contribute to shaping the severity of mastitis, duration of symptoms and resolution of the disease. Similarly, it is increasingly recognised that susceptibility and severity of mastitis in dairy cattle is dependent on complex interactions between microorganisms and the host immune response [17]. A better understanding of the aetiology of mastitis is urgently required, and will assist in the development of improved prevention and treatment strategies.

Mouse models of mastitis involve administration of a mastitis-inducing agent to the mammary gland, usually whilst the mouse is lactating, and examination of the host immune response, and progression and resolution of the disease $[18,19]$. Although the small physical size of mice can pose technical difficulties such as tissue manipulation and sampling, mouse models of disease offer the significant advantage of being time and cost effective 
over larger animal models. The identical genetic profile of inbred mouse strains reduces variability between animals leading to the ability to generate statistically significant results using a low cohort size. Importantly, the excellent availability of genetically modified mice, antibodies to mouse-specific proteins and other experimental approaches such as adoptive cell transfer enables the identification of new cellular and molecular mechanisms, which can spur translational research leading to clinical trials to treat and prevent mastitis in both human and animal species.

There are a number of important variations in the mouse models employed to study mastitis. Some protocols involve weaning of offspring resulting in mammary gland involution, some administer live pathogens and others administer bacterial products that stimulate inflammation in the absence of active infection. The carrier for the mastitis-inducing agent also varies. In this commentary, we discuss the advantages and study design limitations of each mouse model, and highlight the importance of appropriately designed experimental approaches employing physiologically relevant mouse models of mastitis.

\section{Permanent removal of pups at mastitis induction}

Removal of pups from the lactating female at the time of mastitis induction is necessary if the mastitis-inducing agent is prepared in an aqueous solution, as suckling pups are likely to remove it [20], resulting in reduced and variable disease induction. The permanent removal of offspring from a lactating mother is referred to in the literature as "forced weaning", and is an experimental approach used to study the biological process of involution $[21,22]$. Forced weaning results in rapid accumulation of milk in the mammary gland, causing death of alveolar epithelial cells and tissue remodelling, ultimately returning the mammary gland architecture to a nonlactating state [23]. Forced weaning elevates inflammatory mediators in the mammary gland including nuclear factor kappa B [24] and downstream signalling factors such as nitric oxide [25], and there is an influx of macrophages [26,27], all of which are critical components of the cell death and tissue remodelling process.

Forced weaning of offspring at the time of administration of the mastitis-inducing agent complicates the analysis of mastitis induction, as both involve an influx of immune cells to the mammary gland (Figure 1). Forced weaning also limits the utility of the mouse model, once lactation has ceased there is no longer the capacity to study the progression or resolution of the disease in the context of a functional lactating mammary gland, or the impact of the disease on milk supply. Reduced milk supply is a critical feature of mastitis in women [3,4], and most women continue to breastfeed during episodes of mastitis in line with World Health Organization and Academy of Breastfeeding Medicine recommendations
$[2,28]$. On the other hand, forced weaning results in accumulation of milk within the ducts which is not secreted. This may be a desirable component of the model if the study aims to investigate the contribution of rapid weaning or the oversupply of milk, which are predisposing factors [2], on susceptibility to mastitis.

An alternative approach to forced weaning is to return pups to the lactating dam following mastitis induction. However the optimal time point for this, that does not lead to removal of the mastitis-inducing agent by the suckling pups, has not been established. Some of the complications associated with forced weaning can be overcome through the use of appropriate controls that distinguish inflammation associated with involution from signals associated with mastitis [29]. Despite this, the physiological relevance of such studies becomes limited to the acute phase of inflammation over the first 24 to 48 hours.

\section{Administration of different mastitis-inducing agents}

A variety of mastitis-inducing agents are utilised in mouse models of mastitis, including different strains of live bacteria and fungi, such as Escherichia coli, Staphylococcus aureus and Candida krusei, and bacterial products such as endotoxin (an outer membrane lipoprotein in Gram-negative bacteria that elicits a strong inflammatory response; also known as lipopolysaccharide) [16,30-32]. Mastitis is inflammation of the breast that may be infective or non-infective [33-35]. Therefore, the particular agent utilised addresses different questions in disease aetiology - the host immune response to an active bacterial infection (live pathogen) compared to the host immune response to an inflammatory stimulus (bacterial product).

Administration of live bacteria to the mammary gland is employed to investigate the immune response to specific infectious bacterial pathogens [30,36] and the efficacy of experimental vaccines $[37,38]$. These studies often describe the acute response over 24 to 48 hours following mastitis induction and forced weaning $[18,19,30,36]$, although longer term studies in mice with suckling pups have also been described [32,39]. Careful monitoring of mice over the disease time course is essential, as the health of mice administered live bacteria can become severely compromised. For example, an $8^{\circ} \mathrm{C}$ drop in body temperature occurs within 24 hours [36], and death within 48 hours [40] following administration of some strains of $S$. aureus to the lactating mouse mammary gland, indicative of septic shock. Sepsis is a severe systemic inflammatory response to infection followed by an immunosuppressed state, and is associated with multiple organ failure and loss of haemodynamic control [41]. These systemic events are likely to compromise mammary gland immune system function, altering the 


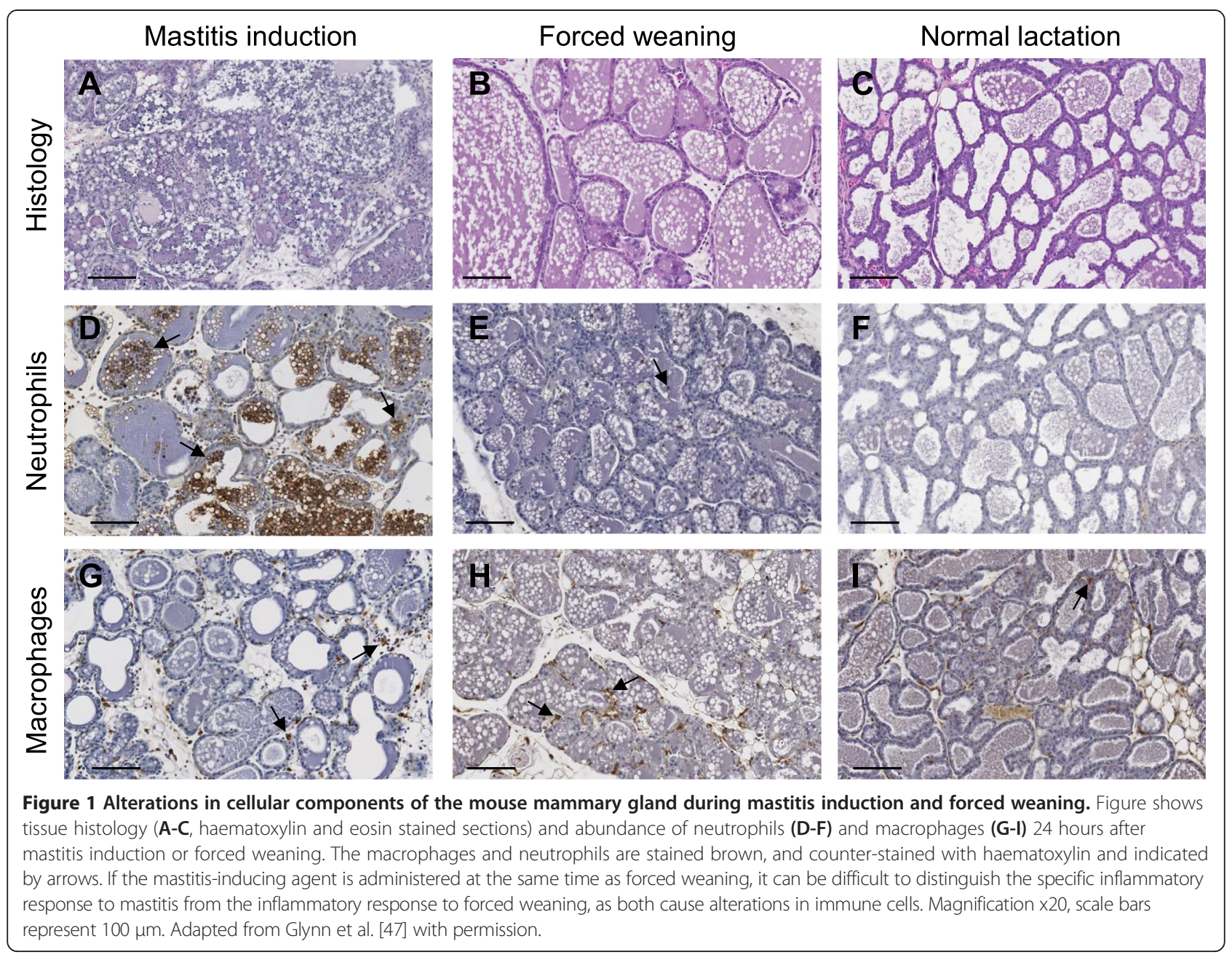

expression of pro-inflammatory and anti-inflammatory cytokines, the survival of immune cells and the capacity for antigen presentation. Although mastitis can lead to sepsis in women [42], this is a rare occurrence and does not replicate mastitis disease progression in the majority of clinical cases.

Compared to administration of live bacteria, administration of bacterial products does not have the same capacity to compromise health of mice, however this approach does not enable the study of how the immune system responds to active bacterial infection. Such studies are best utilised to investigate the host inflammatory response, rather than the invading pathogen. The host inflammatory response, including cells, cytokines and intracellular inflammatory mediators, have been implicated in the severity of mastitis in a number of human $[43,44]$, bovine $[45,46]$ and mouse studies $[16,47,48]$, and the therapeutic benefit of dampening this inflammation is currently being explored in mice [49-52]. Therefore, studies that employ administration of bacterial products rather than live bacteria address how the host immune response affects mastitis disease, and can be utilised to explore potential new therapeutics for further development in pre-clinical and clinical studies.

In addition to variations in the mastitis-inducing agent between different studies, the carrier for the agent also varies. The majority of mouse model studies administer the mastitis-inducing agent in an aqueous carrier such as phosphate-buffered saline [18-20,29-32]. Use of an aqueous carrier poses two physiologically relevant problems: (1) the offspring must be removed from the lactating dam to prevent the offspring from suckling and thus removing the mastitis-inducing agent from the mammary gland as discussed above; and (2) the mastitisinducing agent disperses in the mammary gland causing a widespread mammary gland immune response, that in some instances leads to systemic infection and sepsis. Recently, Matrigel was used as an alternative carrier for deposition of a mastitis-inducing agent into the mammary gland of lactating mice [47]. Matrigel is a 
gelatinous mixture of proteins that is liquid at $4^{\circ} \mathrm{C}$ and becomes solid at body temperature. Bacterial endotoxin was combined with Matrigel on ice, and inserted into the teat canal of lactating mice [47], where it formed a solid plug. This feature of Matrigel has a number of physiologically relevant benefits over traditional aqueous carriers, as the mastitis-inducing agent remains localised to a specific region of the mammary gland, thus enabling the continued suckling of pups and analysis of the full course of disease through to resolution, in the context of a lactating mammary gland.

Further development of mouse models utilising Matrigel as a carrier may provide a number of new avenues for exciting research. The cellular and molecular mechanisms that lead to reduced milk supply associated with mastitis can be explored, as pups can continue to suckle throughout the study. Administration of a live pathogen in Matrigel may be less likely to develop into a systemic infection compared to the same pathogen in an aqueous carrier. In addition, many women cease breastfeeding due to mastitis [3-5], and the impact of milk accumulation in the context of acute mastitis could be investigated. However, there are a number of questions regarding the model that are yet to be addressed, including the utility of the model to study mastitis caused by specific strains of live microorganisms. Furthermore, administration of Matrigel in the absence of endotoxin caused an increase in macrophage abundance after 7 days [47]. This is likely to be part of the host response to remove the plug, however it is unknown whether Matrigel clearance affects the host immune response to the stimulus.

\section{Conclusion}

Utilisation of mouse models of mastitis have the potential to greatly assist in the development of new drug treatments for further testing in clinical trials, and to improve our understanding of the relationships between the microbiome, the host immune response and lactation. However, caution should be applied when incorporating knowledge gained in mouse studies to our overall understanding of mastitis. The use of different types of mastitis-inducing agents and carriers affects the conclusions that can be drawn from the research. The physiological limitations of approaches such as forced weaning and systemic infection must also be considered. Appropriately designed research should employ good controls to delineate the immune response to forced weaning from the immune response to mastitis, and closely monitor the health of the mice. Further development of a mouse model that enables continued suckling of pups and stability of mastitis-inducing agents such as live pathogens and other inflammatory stimuli within the mammary gland will assist in the generation of physiologically relevant new knowledge on the development, progression and resolution of mastitis.

\section{Competing interests}

The authors (WVI, DJG and MRH) have filed a Patent Corporation Treaty (PCT) Patent Application on Treatment and Prevention of Mastitis.

\section{Authors' contributions}

All authors contributed to discussions on manuscript content. WVI wrote the first draft, with further text and editing by DJG and MRH. All authors read and approved the final manuscript.

\section{Authors' information}

All authors hold PhDs in health science, have extensive experience in developing and utilising mouse models of disease, and together they conduct a research program in understanding mastitis, mammary gland inflammation and lactation insufficiency.

\section{Acknowledgements}

WVI is a National Breast Cancer Foundation and The Hospital Research Foundation Fellow, MRH is an Australian Research Council Fellow [ID:110100297].

\section{Author details}

'Discipline of Surgery, School of Medicine, The Queen Elizabeth Hospital, University of Adelaide, Woodville, Australia. ${ }^{2}$ Robinson Research Institute, University of Adelaide, Adelaide, Australia. ${ }^{3}$ Discipline Physiology, School of Medical Sciences, University of Adelaide, Adelaide, Australia.

Received: 24 September 2014 Accepted: 4 March 2015

Published online: 26 March 2015

\section{References}

1. Wambach KA. Lactation mastitis: a descriptive study of the experience. J Hum Lact. 2003;19(1):24-34.

2. Amir LH, Academy of Breastfeeding Medicine Protocol Committee. ABM clinical protocol \#4: Mastitis, revised March 2014. Breastfeed Med. 2014;9(5):239-43.

3. Fetherston C. Characteristics of lactation mastitis in a Western Australian cohort. Breastfeed Rev. 1997;5(2):5-11.

4. Michie C, Lockie F, Lynn W. The challenge of mastitis. Arch Dis Child. 2003;88(9):818-21.

5. Wockel A, Beggel A, Rucke M, Abou-Dakn M, Arck P. Predictors of inflammatory breast diseases during lactation-results of a cohort study. Am J Reprod Immunol. 2010;63(1):28-37.

6. Lunney KM, lliff P, Mutasa K, Ntozini R, Magder LS, Moulton LH, et al. Associations between breast milk viral load, mastitis, exclusive breastfeeding, and postnatal transmission of HIV. Clin Infect Dis. 2010;50(5):762-9.

7. Semba RD. Mastitis and transmission of human immunodeficiency virus through breast milk. Ann N Y Acad Sci. 2000;918:156-62.

8. Osterman KL, Rahm VA. Lactation mastitis: bacterial cultivation of breast milk, symptoms, treatment, and outcome. J Hum Lact. 2000;16(4):297-302.

9. Jonsson S, Pulkkinen MO. Mastitis today: incidence, prevention and treatment. Ann Chir Gynaecol Suppl. 1994;208:84-7.

10. Fetherston C. Mastitis in lactating women: physiology or pathology? Breastfeed Rev. 2001;9(1):5-12.

11. Kvist L, Larsson BW, Hall-Lord ML, Steen A, Schalen C. The role of bacteria in lactational mastitis and some considerations of the use of antibiotic treatment. Int Breastfeed J. 2008;3:6.

12. Delgado S, Arroyo R, Jimenez E, Marin ML, del Campo R, Fernandez L, et al. Staphylococcus epidermidis strains isolated from breast milk of women suffering infectious mastitis: potential virulence traits and resistance to antibiotics. BMC Microbiol. 2009;9:82.

13. Walia HS, Abraham TK, Shaikh H. Fungal mastitis. Case report. Acta Chir Scand. 1987;153(2):133-5.

14. Kvist $L$. Re-examination of old truths: replication of a study to measure the incidence of lactational mastitis in breastfeeding women. Int Breastfeed J. 2013;8:2.

15. Ingman W, Glynn DJ, Hutchinson MR. Inflammatory mediators in mastitis and lactation insufficiency. J Mammary Gland Biol Neoplasia. 2014;19(2):161-7.

16. Zheng J, Watson AD, Kerr DE. Genome-wide expression analysis of lipopolysaccharide-induced mastitis in a mouse model. Infect Immun. 2006;74(3):1907-15. 
17. Thompson-Crispi K, Atalla H, Miglior F, Mallard BA. Bovine mastitis: frontiers in immunogenetics. Front Immunol. 2014;5:493.

18. Chandler RL. Experimental bacterial mastitis in the mouse. J Med Microbiol. 1970;3(2):273-82

19. Anderson JC. Experimental staphylococcal mastitis in the mouse: the induction of chronic mastitis and its response to antibiotic therapy. J Comp Pathol. 1977;87(4):611-21.

20. Brouillette E, Talbot BG, Malouin F. The fibronectin-binding proteins of Staphylococcus aureus may promote mammary gland colonization in a lactating mouse model of mastitis. Infect Immun. 2003;71(4):2292-5.

21. Stein T, Salomonis N, Gusterson BA. Mammary gland involution as a multi-step process. J Mammary Gland Biol Neoplasia. 2007:12(1):25-35.

22. Clarkson RW, Watson CJ. Microarray analysis of the involution switch. J Mammary Gland Biol Neoplasia. 2003;8(3):309-19.

23. Watson CJ. Involution: apoptosis and tissue remodelling that convert the mammary gland from milk factory to a quiescent organ. Breast Cancer Res. 2006;8(2):203.

24. Baxter FO, Came PJ, Abell K, Kedjouar B, Huth M, Rajewsky K, et al. IKKbeta/2 induces TWEAK and apoptosis in mammary epithelial cells. Development. 2006;133(17):3485-94.

25. Zaragoza R, Bosch A, Garcia C, Sandoval J, Serna E, Torres L, et al. Nitric oxide triggers mammary gland involution after weaning: remodelling is delayed but not impaired in mice lacking inducible nitric oxide synthase. Biochem J. 2010;428(3):451-62.

26. O'Brien J, Martinson H, Durand-Rougely C, Schedin P. Macrophages are crucial for epithelial cell death and adipocyte repopulation during mammary gland involution. Development. 2012;139(2):269-75.

27. Stein T, Morris JS, Davies CR, Weber-Hall SJ, Duffy MA, Heath VJ, et al. Involution of the mouse mammary gland is associated with an immune cascade and an acute-phase response, involving LBP, CD14 and STAT3. Breast Cancer Res. 2004;6(2):R75-91.

28. WHO. Mastitis: Causes and Management. Geneva, Switzerland: World Health Organization; 2000.

29. Nazemi S, Aalbaek B, Kjelgaard-Hansen M, Safayi S, Klaerke DA, Knight CH. Expression of acute phase proteins and inflammatory cytokines in mouse mammary gland following Staphylococcus aureus challenge and in response to milk accumulation. J Dairy Res. 2014;81(4):445-54

30. Gonen E, Vallon-Eberhard A, Elazar S, Harmelin A, Brenner O, Rosenshine I, et al. Toll-like receptor 4 is needed to restrict the invasion of Escherichia coli P4 into mammary gland epithelial cells in a murine model of acute mastitis. Cell Microbiol. 2007;9(12):2826-38.

31. Jonsson P, Lindberg M, Haraldsson I, Wadstrom T. Virulence of Staphylococcus aureus in a mouse mastitis model: studies of alpha hemolysin, coagulase, and protein A as possible virulence determinants with protoplast fusion and gene cloning. Infect Immun. 1985;49(3):765-9.

32. Guhad FA, Jensen HE, Aalbaek B, Rycroft A, Hau J. A murine model for the study of mycotic mastitis. J Comp Pathol. 1995;113(4):315-25.

33. Thomsen AC, Espersen T, Maigaard S. Course and treatment of milk stasis, noninfectious inflammation of the breast, and infectious mastitis in nursing women. Am J Obstet Gynecol. 1984;149(5):492-5

34. Thomsen AC, Hansen KB, Moller BR. Leukocyte counts and microbiologic cultivation in the diagnosis of puerperal mastitis. Am J Obstet Gynecol. 1983;146(8):938-41.

35. Kvist $\sqcup$. Toward a clarification of the concept of mastitis as used in empirical studies of breast inflammation during lactation. J Hum Lact. 2010;26(1):53-9.

36. Breyne K, Cool SK, Demon D, Demeyere K, Vandenberghe T, Vandenabeele $P$, et al. Non-classical prolL-1 beta activation during mammary gland infection is pathogen-dependent but caspase-1 independent. PLoS One. 2014;9(8):e105680.

37. Hu C, Gong R, Guo A, Chen H. Protective effect of ligand-binding domain of fibronectin-binding protein on mastitis induced by Staphylococcus aureus in mice. Vaccine. 2010;28(24):4038-44

38. Garcia V, Gomez M, Iglesias M, Sanjuan N, Gherardi M, Cerquetti MC, et al. Intramammary immunization with live-attenuated Staphylococcus aureus: microbiological and immunological studies in a mouse mastitis model. FEMS Immunol Med Microbiol. 1996;14(1):45-51.

39. Tuchscherr LP, Buzzola FR, Alvarez LP, Caccuri RL, Lee JC, Sordelli DO. Capsule-negative Staphylococcus aureus induces chronic experimental mastitis in mice. Infect Immun. 2005:73(12):7932-7.
40. Bramley AJ, Patel AH, O'Reilly M, Foster R, Foster TJ. Roles of alpha-toxin and beta-toxin in virulence of Staphylococcus aureus for the mouse mammary gland. Infect Immun. 1989:57(8):2489-94

41. Doi K, Leelahavanichkul A, Yuen PS, Star RA. Animal models of sepsis and sepsis-induced kidney injury. J Clin Invest. 2009;119(10):2868-78.

42. Demey HE, Hautekeete ML, Buytaert P, Bossaert LL. Mastitis and toxic shock syndrome. Acta Obstet Gynecol Scand. 1989;68(1):87-8.

43. Fetherston CM, Lai CT, Hartmann PE. Relationships between symptoms and changes in breast physiology during lactation mastitis. Breastfeed Med. 2006;1(3):136-45

44. Fetherston CM, Wells JI, Hartmann PE. Severity of mastitis symptoms as a predictor of $\mathrm{C}$-reactive protein in milk and blood during lactation. Breastfeed Med. 2006:1(3):127-35.

45. Thompson-Crispi KA, Miglior F, Mallard BA. Incidence rates of clinical mastitis among Canadian Holsteins classified as high, average, or low immune responders. Clin Vaccine Immunol. 2013;20(1):106-12.

46. Catalani E, Amadori M, Vitali A, Lacetera N. Short communication: Lymphoproliferative response to lipopolysaccharide and incidence of infections in periparturient dairy cows. J Dairy Sci. 2013;96(11):7077-81.

47. Glynn DJ, Hutchinson MR, Ingman W. Toll-like receptor 4 regulates lipopolysaccharide-induced inflammation and lactation insufficiency in a mouse model of mastitis. Biol Reprod. 2014;90(5):91.

48. Elazar S, Gonen E, Livneh-Kol A, Rosenshine I, Shpigel NY. Essential role of neutrophils but not mammary alveolar macrophages in a murine model of acute Escherichia coli mastitis. Vet Res. 2010;41(4):53.

49. Li D, Fu Y, Zhang W, Su G, Liu B, Guo M, et al. Salidroside attenuates inflammatory responses by suppressing nuclear factor-kappaB and mitogen activated protein kinases activation in lipopolysaccharide-induced mastitis in mice. Inflamm Res. 2013;62(1):9-15.

50. Fu Y, Gao R, Cao Y, Guo M, Wei Z, Zhou E, et al. Curcumin attenuates inflammatory responses by suppressing TLR4-mediated NF-kappaB signaling pathway in lipopolysaccharide-induced mastitis in mice. Int Immunopharmacol 2014;20(1):54-8.

51. Ruifeng G, Yunhe F, Zhengkai W, Ershun Z, Yimeng L, Minjun Y, et al. Chlorogenic acid attenuates lipopolysaccharide-induced mice mastitis by suppressing TLR4-mediated NF-kappaB signaling pathway. Eur J Pharmacol. 2014;729:54-8.

52. Chen $\mathrm{H}, \mathrm{Mo} X, \mathrm{Yu} J$, Huang Z. Alpinetin attenuates inflammatory responses by interfering toll-like receptor 4/nuclear factor kappa B signaling pathway in lipopolysaccharide-induced mastitis in mice. Int Immunopharmacol. 2013;17(1):26-32

\section{Submit your next manuscript to BioMed Central and take full advantage of:}

- Convenient online submission

- Thorough peer review

- No space constraints or color figure charges

- Immediate publication on acceptance

- Inclusion in PubMed, CAS, Scopus and Google Scholar

- Research which is freely available for redistribution 